\title{
Design and Construction of a $\sim 7 \times$ Low-Concentration Photovoltaic System Based on Compound Parabolic Concentrators
}

\author{
Mark A. Schuetz, Member, IEEE, Kara A. Shell, Scott A. Brown, Gregory S. Reinbolt, \\ Roger H. French, Member, IEEE, and Robert J. Davis, Member, IEEE
}

\begin{abstract}
We report on the design, construction, and initial performance measurements of a low-concentration photovoltaic system based on compound parabolic concentrators (CPCs). The system is approximately a $7 \times$ concentration system and uses commercially available laser groove buried contact monocrystalline silicon photovoltaic cells. The CPCs are fabricated using a secondsurface aluminized acrylic mirror with proven weather durability. The asymmetric CPC optical design was driven by a balance between concentration factor, thermal issues, and optical angle of acceptance and was thoroughly evaluated by optical ray tracing. The design was targeted for a single-axis tracking system, with extruded aluminum heat sinks doubling as structural components. We fabricated a 120 -cell $(10 \times 12)$ prototype array, and over three months of operation, we estimated an approximate peak total system power efficiency of $\mathbf{7 . 9 \%}$, limited mostly by the CPC optical efficiency $(\sim 55 \%)$ and the cell conversion efficiency. We discuss several issues regarding system performance, reliability, and cost.
\end{abstract}

Index Terms-Compound parabolic concentrators (CPCs), concentrated photovoltaic (CPV), low-concentration photovoltaics (LCPV).

\section{INTRODUCTION}

$\mathbf{R}$ ECENTLY, there has been a resurgence in interest in photovoltaic systems of low solar concentration [roughly $<20 \times$ solar concentration factor (CF)] [1]-[5]. Such systems are potentially low-cost photovoltaic (PV) systems, since they reduce greatly the extensive amount of pure semiconductor material needed in unconcentrated PV modules while avoiding the need for expensive and complex optical and thermal management systems required for high concentration $(>400 \times)$ systems.

Manuscript received July 11, 2011; revised November 2, 2011; accepted December 18, 2011. Date of publication April 9, 2012; date of current version June 18, 2012. This work was supported in part by the Third Frontier Program of the Ohio Department of Development under Grant 10-416 and in part by the Ohio Wright Center for Photovoltaics Innovation and Commercialization.

M. A. Schuetz, K. A. Shell, S. A. Brown, and G. S. Reinbolt are with Replex Plastics, Mt. Vernon, OH 43050 USA (e-mail: mark@replex.com; kara@replex.com; scott@ replex.com; greg@ replex.com).

R. H. French is with the Department of Materials Science and Engineering, Case Western Reserve University, Cleveland, OH 44106 USA (e-mail: rxf131@case.edu).

R. J. Davis is with the Nanotech West Lab and the Institute for Materials Research, The Ohio State University, Columbus, OH 43212 USA (e-mail: davis.2316@osu.edu).

Color versions of one or more of the figures in this paper are available online at http://ieeexplore.ieee.org.

Digital Object Identifier 10.1109/JPHOTOV.2012.2186283
Moreover, unlike high-concentration photovoltaic (HCPV) systems, which generally have tracking requirements of on the order of one angular degree, low-concentration photovoltaic (LCPV) designs can retain a high angle of optical acceptance, with the possibility of using much less expensive trackers and lightweight structural components. Such systems would also be deployable in mid-northern latitudes, where HCPV systems are not likely to be economical due to the lower direct normal irradiance (DNI) climate. Finally, the low CFs of LCPV systems also permit a wider range of materials to be used in their construction (encapsulants, optics, etc.) without concerns for solar degradation in an HCPV environment.

This paper describes the design, construction, and testing of a prototype LCPV system, which we refer to as "Gen 1." The system consists of three primary parts: the optics, the cell and its encapsulation, and a heat sink. The latter serves triple duty as a heat dissipation unit, an overall mechanical skeleton for the system, and an optical alignment component. The overall system dimensions were chosen to be $1.6 \times 1.0 \mathrm{~m}$, matching a popular size of unconcentrated (" $1 \times$ ") panels and was designed for inclined single-axis tracking.

\section{OPTICS}

Numerous factors influenced our selection of the compound parabolic concentrators (CPC) optical design for the prototype array. These factors included 1) the desire for an optical acceptance half-angle of $23.5^{\circ}$ in the untracked direction to allow for seasonal variations in the angle of the sun without the need for system mechanical adjustment; 2) the geometries of commercially available silicon cells; 3 ) the heat load that the CPC would impose on economical, passive heat dissipation solutions, with an engineering target chosen of $T_{\text {cell }}$ raising no more than $10^{\circ} \mathrm{C}$ above ambient temperature under AM1.5D illumination conditions; and 4) fabrication limitations of the thermoforming process used to manufacture the CPCs.

We chose an asymmetric CPC design of 1.33:1 (width/length ratio) with an overall geometric $\mathrm{CF}$ of $\sim 10.3$, with mirror losses resulting in an overall CPC CF of 7. The base design is also truncated to a height of $143 \mathrm{~mm}$, approximately $75 \%$ of the height of a fully developed CPC for the nontracking direction [6], which optical ray tracing suggested was at very little cost to CF. The final Gen 1 prototype, however, also contained some slightly shorter CPCs that confirmed the insensitivity of power to height in this size range. 


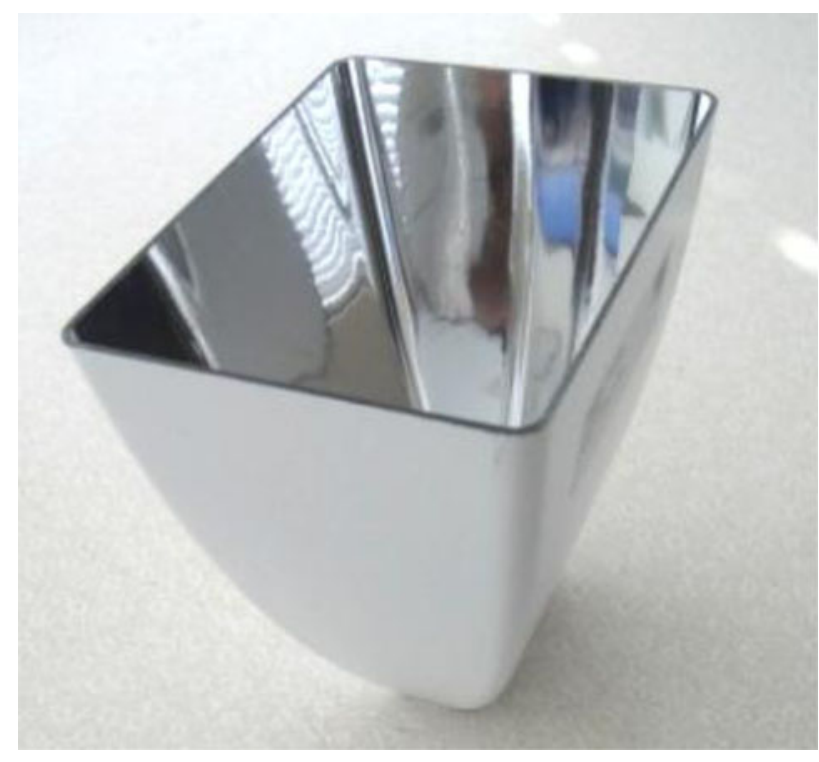

Fig. 1. Completed CPC used in this prototype construction.

CPCs were fabricated by thermoforming mirror-grade acrylic material over a syntactic foam mold, followed by a thermal evaporation of approximately $100 \mathrm{~nm}$ of aluminum to form a second-surface mirror. A protective coating is then applied to the aluminum to provide weather durability; this particular mirror product has been sold into the transportation sector for over a decade with exceptional product lifetimes in weather. This second-surface mirror process results in an $86 \%$ overall visible optical power reflectivity with a lower wavelength cutoff of approximately $375 \mathrm{~nm}$. A picture of a finished Gen $1 \mathrm{CPC}$ is shown in Fig. 1.

Optical ray tracing computer simulations [7] for the CPC designs were utilized throughout the design process. The illumination model for the ray tracing assumed DNI with randomly placed rays of $546 \mathrm{~nm}$ wavelength at a uniform intensity of $1000 \mathrm{~W} / \mathrm{m}^{2}$. At least 10000 rays were typically traced for a design. Uniformity of the concentrated light at the exit aperture was surprisingly sensitive to factors such as the radii of the corners of the CPC and the thickness of the acrylic. Ray tracing of the final Gen 1 CPC design (see Fig. 2) suggests the presence of local hotspots of $\sim 26 \times$ concentration of a few millimeters size at the exit aperture, the presence of which were subsequently confirmed in the observed illumination patterns. The ray tracing also suggested that a significant fraction of the rays encountered 1-2 bounces off of the CPC mirror on their path to the exit aperture, and that a significant amount of optical energy at the exit aperture was significantly off of the illumination axis. On average, the simulations predicted $6.879 \mathrm{~kW} / \mathrm{m}^{2}$ at the exit, and more details and discussion of the optical ray tracing results will be published elsewhere [8].

The base CPC design resulted in a plan for 10 strings of 12 units each for the prototype, the backbone of each string being a continuous 1.6-m length of heat sink.

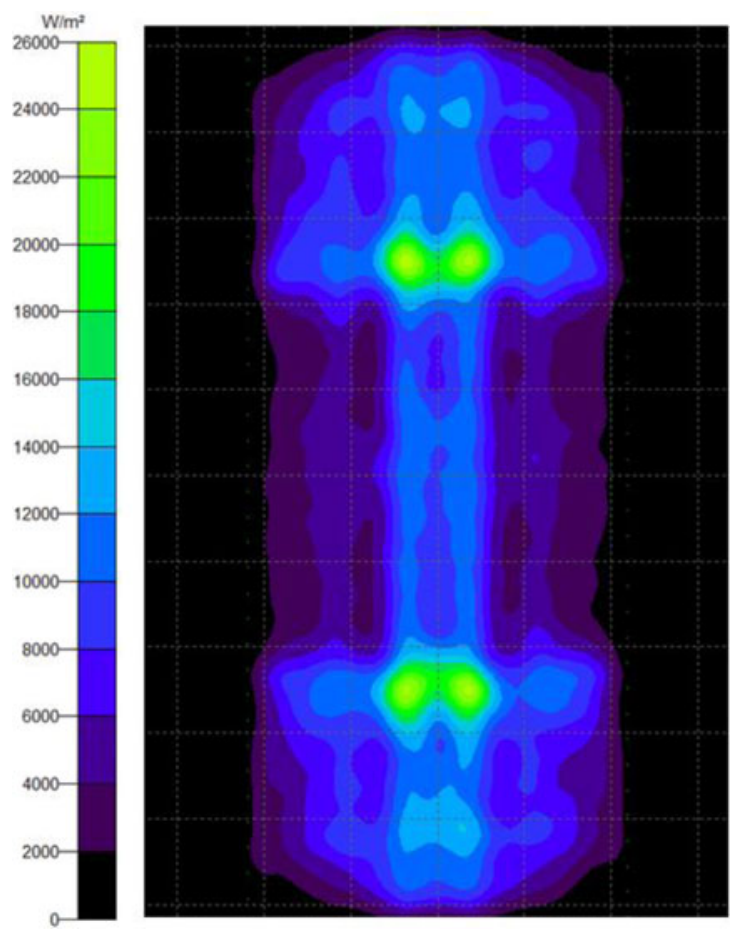

Fig. 2. Optical ray tracing results of the final CPC design for $1000 \mathrm{~W} / \mathrm{m}^{2}$ of 546-nm-wavelength illumination. The graph shows predicted optical intensity at the exit aperture in Watts per meter squared. The optical nonuniformities of the CPC were qualitatively verified visually in on-sun, on-cell conditions.

\section{CELLS}

In conjunction with the CPC design, unpackaged bare c-Si PV cells were obtained commercially [9]. The $\mathrm{n}^{+}$on $\mathrm{p}$ cells were laser groove buried contact cells with an AM1.5D base technology conversion efficiency of $17-18 \%$. For this application, to reduce series resistance losses the front finger widths were widened during fabrication in anticipation of being used in LCPV, with the result that shading losses reduced the cell conversion efficiencies at $1 \times$ to approximately $16-17 \%$. The cell dimensions of $22 \times 53 \mathrm{~mm}$ made efficient use of the $125 \mathrm{~mm}$ $\times 125 \mathrm{~mm}$ wafer size used by the vendor, and included busbars of metal for interconnection that would subsequently be located outside of the illumination area. The bus bars ran the long length of the cell to minimize series resistance losses. No particular attempt was made to match cells for current in strings.

\section{HEAT SINK}

As mentioned previously, a design goal of the heat sink was to passively cool the cell to within $10{ }^{\circ} \mathrm{C}$ of ambient temperature under the final concentrating illumination conditions in static (windless) air. We designed and modeled (via finite element analysis) a nine-fin heat sink that could be readily extruded from aluminum into long sections (see Fig. 3). Since much of the design of the heat sink and the optical elements were done in parallel, the heat sink design and modeling assumed $10 \times$ concentration and $15 \%$ cell conversion efficiency and still air (no wind). Modeling suggested that temperature rise at the cell would be no more than $\sim 6^{\circ} \mathrm{C}$ under these conditions 


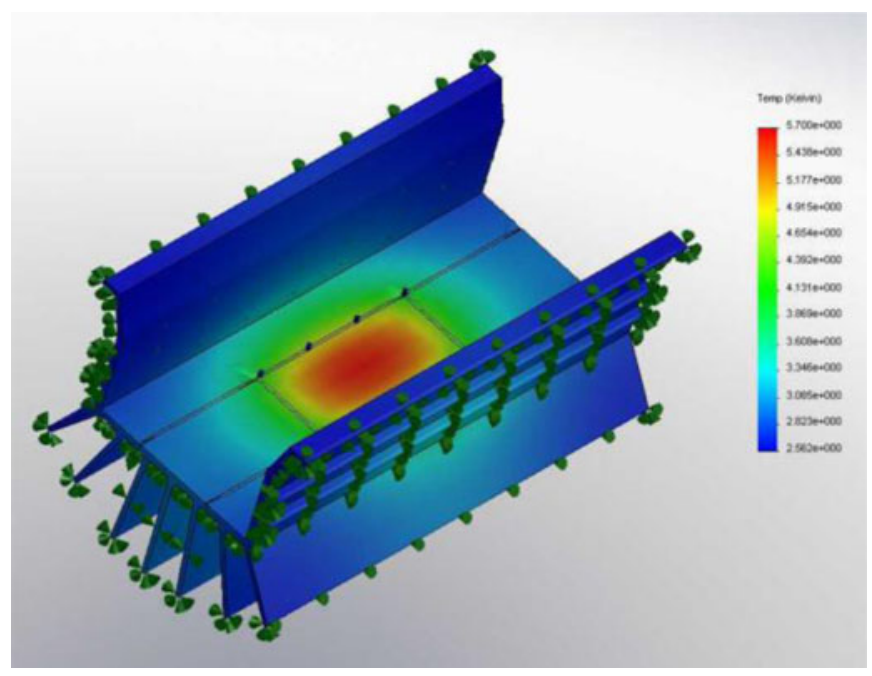

Fig 3. Finite-element modeling temperature rise results for the heat sink design based on $10 \times$ illumination and $15 \%$ cell efficiency assumptions. Still air (no wind) was also assumed as a worst-case condition.

(see Fig. 3) given the properties of the boron-nitride-filled adhesive pads (Parker Therm-a-Gap Type 974, thermal conductivity $6.0 \mathrm{~W} /(\mathrm{m} \cdot \mathrm{K})$, dielectric strength $5.1 \mathrm{kV} / \mathrm{mm})$ that we planned to use to provide good thermal contact between the cells and the heat sinks. The pads also buffered the differences in the temperature coefficients of expansion of the aluminum heat sink and the silicon cell, which under constant thermal cycling could result in cell cracking. The two upper fins also provided a contact surface for attachment of the CPCs and were given additional ribs for mechanical reinforcement. We verified the performance of the heat sink separately in a lab experiment using electrical heating elements and thermocouples.

\section{COnstruction}

Prior to the construction of the entire prototype, we tested a single cell mounted on a short heat sink, in turn mounted on a tiltable test stand, with and without a CPC. This was to test the overall cell $I-V$ performance under illumination and to reduce our concerns that the CPC illumination nonuniformities found in the optical ray tracing might have a severely detrimental effect on system performance. The $I-V$ results for a singlecell/CPC heat sink unit under $\sim 800 \mathrm{~W} / \mathrm{m}^{2}$ bright outdoor sun illumination are shown in Fig. 4. Since we could take $I-V$ scans very quickly (only a few seconds per scan) with and without the CPC, illumination conditions were approximately the same for both curves, which was also confirmed using an optical pyranometer mounted on the stand.

Comparison of the $I-V$ curves indicates typical performance of a CPV system. Short-circuit current, $I_{\mathrm{sc}}$, increased dramatically from 0.45 to $2.53 \mathrm{~A}$, which is a $5.6 \times$ increase, in line with our expectations of our optics design, but less than the $\sim 6.8 \times$ predicted by our idealized optical ray tracing. Open-circuit voltage $V_{\text {oc }}$ increased from 566 to $626 \mathrm{mV}$ under concentration, in rough agreement with the expected increase of $n k T \ln (\mathrm{CF})$. While fill factor increased slightly, i.e., from $66.8 \%$ to $69.6 \%$

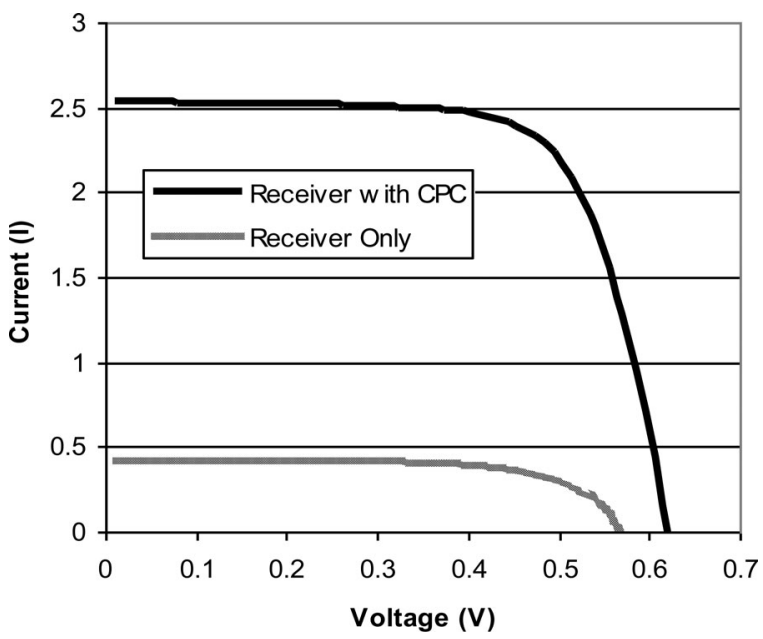

Fig. 4. $I-V$ results of a single cell/heat sink unit with and without a CPC. These $I-V$ scans were taken in outdoor direct sun at $\sim 800 \mathrm{~W} / \mathrm{m}^{2}$ illumination in midday under cloudless skies.

under concentration, however, the total conversion efficiency of the unit on an area basis dropped from $15 \%(1 \times$, without CPC $)$ to $7.9 \%$, mostly due to the predicted optical losses in the CPC. Meanwhile, however, intentional misdirection of the test stand from the sun confirmed our expectations of the optical acceptance angles of the CPCs as outlined in Section II, with the performance of a module falling off quickly only when intentionally misaimed more than $\pm 20^{\circ}$ in the untracked direction.

We then proceeded to build the Gen 1 prototype. Three initial strings of 12 units each were built with the CPCs outlined earlier, and the remaining seven strings were built with very slightly shortened CPCs, due to manufacturing issues, that had essentially identical performance. Each string contained its own blocking diode and junction box. Individual cells on a string were tabbed in series and were encapsulated in a precommercial DuPont ionomer material with a process temperature of $130{ }^{\circ} \mathrm{C}$ using a vacuum bag process, and all but one string receiving a cover glass directly over the cell. We used untempered, regular sheet glass since PV glass was not readily available in the unusual size needed for this system. During string fabrication and assembly, a small thermoforming problem led us to reduce the CPC height slightly for all but the first 36 modules, with a negligible effect on module output power.

\section{InStallation AND PeRformance}

While this prototype was designed for an inclined single-axis tracker, a dual-axis tracker was available to us for testing so we used it for this testing. The strings were mounted on crossbrackets, which were in turn attached to the tracker. Connecting the ten strings in parallel proved tedious and is an obvious area for improvement in future designs. Fig. 5 shows the completed prototype; the three strings to the right have slightly taller CPCs that were fabricated first.

Fig. 6 shows the dc power output of the system on a bright sunny day in late fall in Central Ohio. In general, in ideal sun conditions, the system demonstrated a total $7.9 \%$ conversion 


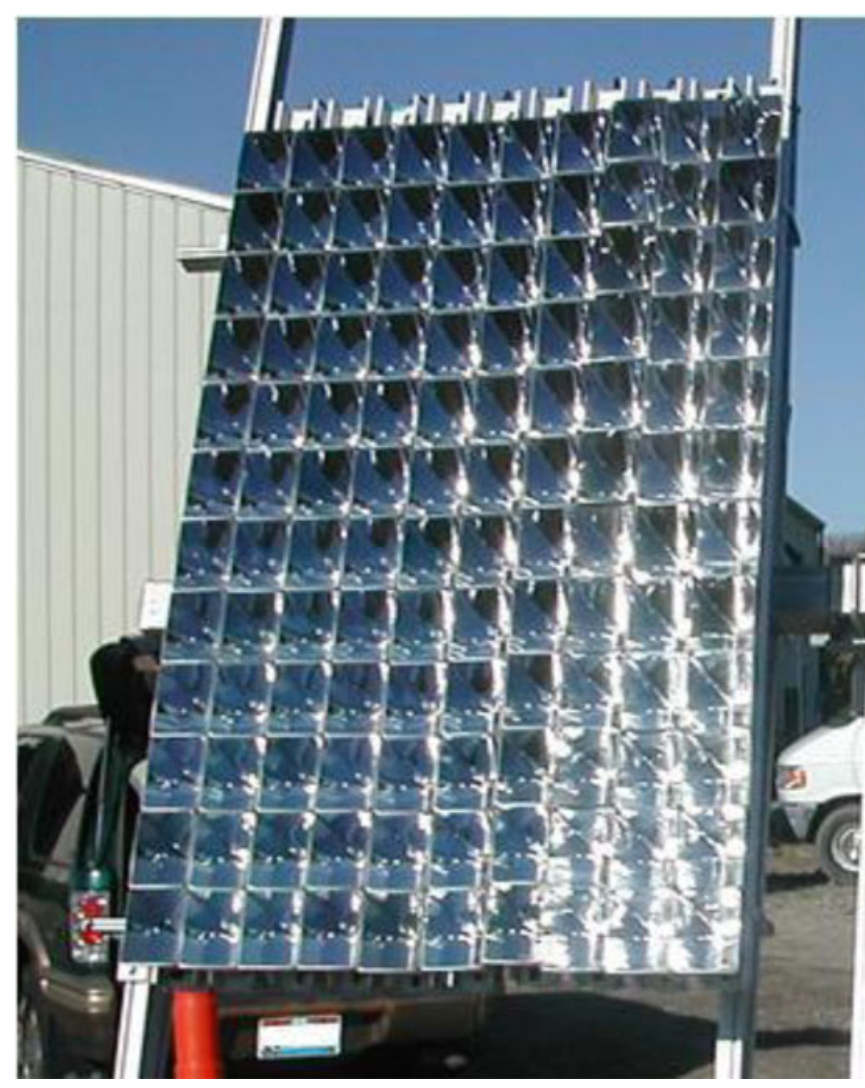

Fig. 5. The completed Gen 1 prototype system, before mounting on the tracker. The three strings on the right were constructed with slightly taller CPCs that were subsequently reduced in height due to thermoforming issues. The small height difference had a negligible impact on power out differences among the strings.

efficiency (only coincidentally the same efficiency number as the single module tested in Section V). This was confirmed in comparison with two commercial, unconcentrated panels located on the same tracker. Analyzing system performance, we can write the total system efficiency $\eta_{\text {total }}$ as

$$
\eta_{\text {total }}=\left(\eta_{\text {opt }}\right)\left(\eta_{\text {elect }}\right)\left(\eta_{\text {cell }}\right)
$$

where $\eta_{\text {opt }}, \eta_{\text {elect }}$, and $\eta_{\text {cell }}$ are the optical, electrical, and cell efficiencies, respectively. Using the estimate of our electrical interconnect losses as $3.64 \%$, and assuming $\eta_{\text {cell }}$ to be approximately $16 \%$, this suggests that our optical efficiency is approximately $51 \%$.

We attribute the primary optical losses in the system to three main causes. For one, as mentioned in Section II, optical ray tracing suggests that a large number of photons reflect twice on their way down to the exit aperture. With a second-surface mirror of $\sim 86 \%$ reflectivity, this is a major cause of optical loss and clearly suggests the potential importance of a first-surface mirror, if one can be made durable. Second, the ray tracing assumes a $100 \%$ absorber at the exit aperture, and the textured AR-coated surface of the cell used here cannot achieve this. The large amount of off-axis illumination at the exit aperture currently limits our ability to estimate this loss. Finally, we directly observed optical power loss due to light leaking from

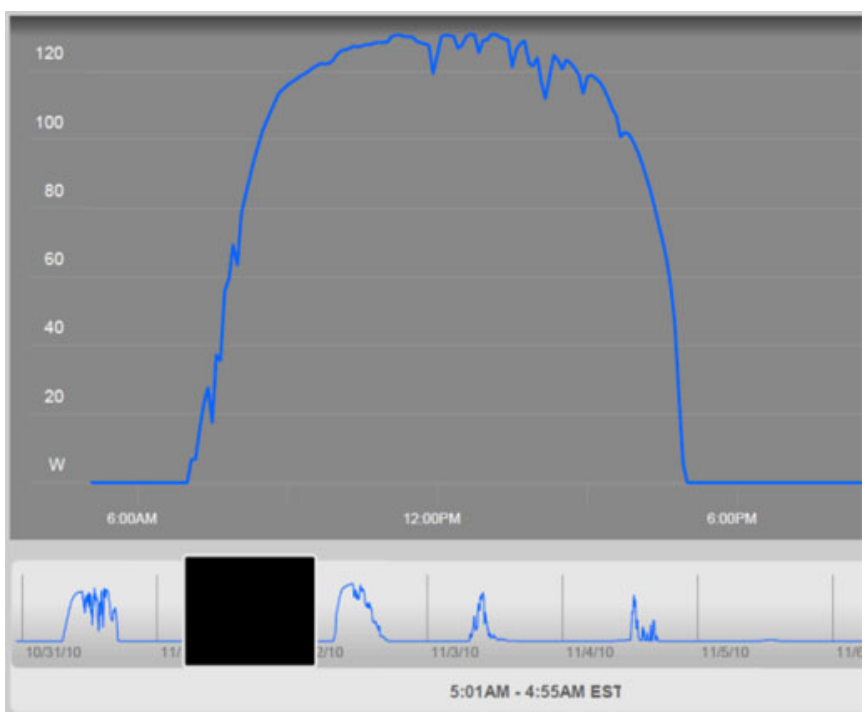

Fig. 6. Typical output of the completed Gen 1 prototype system on a bright sunny day in late fall in Athens, $\mathrm{OH}$.

the modules in the plane of the cover glass, a leakage that is also in part attributable to off-axis effects.

Somewhat surprising in both the single-cell and the full system results for Gen 1 was the lack of an obvious negative effect of the optical uniformity, as predicted by the ray tracing, on power output. This is likely due to two reasons. First, the ray tracing results are for single-wavelength light with no account for light scattering at the acrylic/air surface; a full spectrum of wavelengths combined with a physical model of roughness of the acrylic surface would tend to smooth out the hotspots that are so prominent in Fig. 2. Second, the relatively long minority carrier lifetimes and long carrier diffusion lengths of silicon would also serve to redistribute carriers from hotspots, smoothing the 2-D minority carrier distribution across the front grid. Nonetheless, this is an interesting topic for simulations and/or future experiments.

Two issues preclude the need here, however, for a more exhaustive description and comparison of the Gen 1 LCPV system power output.

First, the optical tracker used for this demonstration would often get lost during less-than-optimum days. This most often resulted in the optical system sensor locking on to a bright cloud while the sun was obscured. After that event, the tracker would slew to its home position and then attempt to once again find the sun, and this process could waste as much as 20 min time in misalignment. The integration of a much more robust tracking solution will be necessary for a new design.

Second, many strings suffered cracked and/or broken cover glass in the first three months in the field. This was due to a combination of our use of untempered glass in the encapsulation, the geometry of the heat sink, mechanical stresses built up in the ionomer encapsulation process, and temperature cycling. The first three months of operation were winter months in Ohio, but we are uncertain if summer operation would be less harsh. The power outputs of strings that suffered cracked glass dropped steeply due to additional optical reflection and water and dirt that accumulated in the cracks. 
Finally, we make two comments on the system design, primarily regarding cost. First, cell efficiency is still a large factor in system performance here, even in low concentration. Using cells of over $20 \%$ efficiency would improve system power output by nearly $25 \%$, likely justifying the use of more complicated and expensive back-contact [2] or other cell designs. Second, our heat sink design performed quite well as suggested by cell efficiency measurements, which is understandable, considering that we designed it for as much as $10 \times$ concentration in still air. However, our cost estimates suggest that for a target system price of $\$ 1.00$ per peak Watt output power, this heat sink alone would consume most of the budget for materials. Hence, a radically simpler, less expensive heat dissipation solution is needed if this type of system could become commercially successful in future projections of the PV market.

\section{CONCLUSION}

We have designed, built, and tested a prototype $\sim 7 \times$ lowconcentration photovoltaic array based on asymmetric compound parabolic concentrator optical elements and commercially available monocrystalline silicon photovoltaic elements. Optical losses in the CPCs and CPC/cover glass/cell interfaces are dominant sources of power loss. An initial set of three months of in-weather data has revealed performance and reliability issues that we will solve or improve upon in a second design.

\section{ACKNOWLEDGMENT}

The authors acknowledge the help of the Edison Welding Institute, Columbus, $\mathrm{OH}$, in the Gen 1 electrical interconnections and the assistance of Dovetail Solar and Wind, Athens, OH, for the installation of the Gen 1 prototype.

\section{REFERENCES}

[1] S. Hatwaambo, H. Hakansson, J. Nilsson, and B. Karlsson, "Angular characterization of low concentrating PV-CPC using low-cost reflectors," Sol. Energy Mater. Sol. Cells, vol. 92, pp. 1347-1351, 2008.

[2] M. M. Bunea, K. W. Johnston, C. M. Bonner, P. Cousins, D. D. Smith, D H. Rose, W. P. Mulligan, and R. M. Swanson, "Simulation and characterization of high efficiency back contact cells for low concentration photovoltaics," in Proc. 35th IEEE Photovoltaic Spec. Conf., 2010, pp. 823-826.

[3] D. C. Walter, V. Everett, A. Blakers, M. Vivar, J. Harvey, J. Muric-Nesic, T. Ratcliff, S. Surve, R. Van Scheppingen, P. Le Lievre, M. Greaves, and A. Tanner, "A 20-sun hybrid PV/thermal linear microconcentrator system for urban rooftop applications," in Proc. 35th IEEE Photovoltaic Spec. Conf., 2010, pp. 831-836.

[4] T. K. Mallick and P. C. Eames, "Design and fabrication of low concentrating second generation PRIDE concentrator," Sol. Energy Mater. Sol. Cells, vol. 91, pp. 597-608, 2007.

[5] A. Luque, G. Sala, G. L. Araugo, and T. Bruton,, "Cost reducing potential of photovoltaic concentration,” Int. J. Sol. Energy, vol. 17, pp. 179-198, 1995.

[6] J. J. O'Gallagher, Non-Imaging Optics in Solar Energy. San Rafael, CA: Morgan \& Claypool, 2008, pp. 7-20.

[7] FEA Using Software From SolidWorks, Concord, MA; Optical Ray Tracing Software Using TracePro, Lambda Res., Littleton, MA.

[8] K. A. Shell et al., "Design and performance of a low-cost acrylic reflector for a $\sim 7 \mathrm{x}$ concentrating photovoltaic module," Proc. SPIE, vol. 8108, pp. 81080A, 2011

[9] Cells were obtained from Narec, Inc., Newcastle, U.K.
Mark A. Schuetz (M'11) received the B.S. and M.S. degrees in mechanical engineering from the Massachusetts Institute of Technology, Cambridge.

He founded Replex Plastics, Mt. Vernon, OH, in 1991, where he is currently the President. He was the Vice President of operations with Plaskolite, Columbus, OH, from 1986 to 1990, an Advanced Engineer with Owens-Corning Fiberglas, Granville, OH, from 1982 to 1986, and a Project Engineer with Advanced Mechanical Technology, Newton, MA, from 1979 to 1981 . He is interested in alternative energy, especially in concentrating photovoltaics, the durability and reliability of photovoltaic systems and materials, and the economics of photovoltaics.

Kara A. Shell received the B.S. (magna cum laude) and M.S. degrees in mechanical engineering from The Ohio State University (OSU), Columbus, in 2008 and 2010, respectively, during which she was heavily involved in the OSU Solar Decathlon entry.

She is currently a Project Engineer with Replex Plastics, Mt. Vernon, OH, where she is engaged in the use of such advanced tools as 3-D computeraided design (CAD) modeling, integrated $\mathrm{CAD} /$ computer-aided manufacturing programming, finite element analysis, and optical ray tracing, including the modeling of compound parabolic concentrators and heat sinks for this project. She is involved in all aspects of product design processes at Replex, from concept to prototype construction to the design and execution of experiments to evaluate prototypes.

Scott A. Brown received the A.A.S. degree in electronics engineering technology from Columbus State Community College, Columbus, OH, in 1994. He has been with Replex Plastics, Mt. Vernon, OH, as a Manufacturing/Project Engineer since 2009. He has more than 13 years of experience in designing manufacturing processes and equipment, thermoforming, vacuum metalizing, and coatings (wet-applied and plasma-enhanced chemical vapor deposition). His work has been focused on concentrator design and manufacturing, concentrated photovoltaic material and system reliability/durability, and electrical interconnect design.

Gregory S. Reinbolt received the A.A.S. degree in polymer color technology/plastics processing. from Terra Community College, Fremont, $\mathrm{OH}$.

He is currently a Process Technician with Replex Plastics, Mt. Vernon, $\mathrm{OH}$ He has been with Replex for 4 years as their Primary Color Technician and has also spent much of the last year at the Ohio State Nanotech West Lab, where he was involved in the design, fabrication, and testing of silicon photovoltaic cells for this project.

Roger H. French (M'07) received the Ph.D. degree in materials science from the Massachusetts Institute of Technology, Cambridge.

$\mathrm{He}$ is currently the F. Alex Nason Professor with the Department of Materials Science and Engineering, Case Western Reserve University (CWRU), Cleveland, OH. He joined CWRU in August 2010 after 24 years of conducting basic research and product development with DuPont's Central Research. $\mathrm{He}$ has broad experience in developing and commercializing optical materials for many different applications and in optimizing these materials for improved radiation durability and lifetime. He is also a nationally recognized expert in Lifetime and Degradation Science for commercial applications, evidenced by his research on attenuating phase shift photomasks, fluoropolymer pellicles for photolithography, immersion lithography imaging fluids, and materials for concentrating photovoltaics projects at DuPont. He has 22 issued patents and more than 145 publications.

Robert J. Davis (M'90) received the Ph.D. degree in applied physics from Cornell University, Ithaca, NY, in 1990.

$\mathrm{He}$ is currently the Director of the Nanotech West Lab, Co-Director of the Ohio Wright Center for Photovoltaics Innovation and Commercialization, Associate Director of the Institute for Materials Research, and an Adjunct Associate Professor of electrical and computer engineering, all with The Ohio State University, Columbus. Prior to Ohio State University, he was with the Process Engineering Group of TriQuint Semiconductor, Dallas, TX, from 2000 to 2005 and a Graduate Faculty Member with the Engineering Science and Mechanics Department, Penn State University, University Park, from 1994 to 2000. He has also been a Visiting Scientist with the IBM Thomas J. Watson Research Center, Yorktown Heights, NY, and the Max-Planck Institute for Solid State Physics. He is the author or co-author of approximately 30 publications and numerous conference proceedings in the areas of electronic materials and processing and solid-state device fabrication.

Dr. Davis is a member of the American Physical Society. 